\title{
On mean field glassy dynamics out of equilibrium
}

\author{
Silvio Franz and Marc Mézard \\ Laboratoire de Physique Théorique de l'Ecole Normale Supérieure
}

\begin{abstract}
We study the off equilibrium dynamics of a mean field disordered systems which can be interpreted both as a long range interaction spin glass and as a particle in a random potential. The statics of this problem is well known and exhibits a low temperature spin glass phase with continuous replica symmetry breaking. We study the equations of off equilibrium dynamics with analytical and numerical methods. In the spin glass phase, we find that the usual equilibrium dynamics (observed when the observation time is much smaller than the waiting time) coexists with an aging regime. In this aging regime, we propose a solution implying a hierarchy of crossovers between the observation time and the waiting time.
\end{abstract}

LPTENS preprint $94 / 05$

cond-mat/9403004

\footnotetext{
${ }^{1}$ Unité propre du CNRS, associée à l'Ecole Normale Supérieure et à l'Université de Paris Sud, 24 rue Lhomond, 75231 Paris Cedex 05, France
} 


\section{Introduction}

A lot of efforts have been devoted in the last fifteen years to the study of equilibrium static and dynamic properties of spin glasses [1, 2, 3]. Comparatively, the off equilibrium dynamical effects have received less attention. The recent years have seen a renewal of interest for this OED. One reason is experimental. While it is clear that many experimental observations are inherently dynamical effects, the status of the off equilibrium dynamical effects have turned recently from that of an annoying perturbance to that of a very powerful probe. Some of the most interesting recent experimental findings in spin glasses, like the slow relaxation of the thermoremanent magnetization, aging, and memory effects during temperature cycling experiments, are inherently out of equilibrium phenomena [4, 5, 6, 7]. Several phenomenological models of these effects have already been proposed, based on ideas of droplets [8, 9] or phase space traps with a broad distribution of trapping times [10. The second origin of this upsurge of interest comes from the theoretical side. Prompted by the experimental observations, it has been realized recently that some microscopic analytical approach to these problems is possible, and that the off equilibrium nature of the dynamics might even cure some old problems of the dynamical approach. The first works on spin glass dynamics, following the idea that the use of a dynamical generating functional could be an alternative to the introduction of replicas [11], focused on the ED [12, 13]. Early attempts to model some aspects of the OED along these same lines have concentrated on the mean field theory of spin glasses close to the critical temperature, taking into account explicitely the changes in external parameters like temperature or magnetic field [14, 15]. More recently, it has been observed that these effects can be studied without any reference to time variation of the external parameters, but by keeping into account the existence of an initial time for the dynamics (corresponding to the quench into the spin glass phase in the experiments), and the existence of a finite waiting time [16, 17, 18, 19, 20].

In this paper we study the off equilibrium dynamics (OED) through a microscopic approach along the lines above. We consider the problem of an oriented $\mathrm{D}$ dimensional manifold embedded in a $\mathrm{D}+\mathrm{N}$ dimensional space, in presence of a random potential. This is a very interesting and general problem [21] which is connected to interface pinning by impurities, directed polymers in disordered media, vortex pinning in high temperature superconductors [22, 23], and also, after various mappings, to growth phenomena [24] or turbulence [25]. We shall work in the limit of an infinite dimensional embedding space $(N \rightarrow \infty)$. This limit has two major advantages. It allows for the derivation of exact integrodifferential equations for the correlation and response functions. Also in this limit the static properties have been studied in details using the replica method, and it has been shown that a full hierarchical replica symmetry breaking (r.s.b.) is needed in order to describe the system [26].

Our work has two aspects. One is an analytic study of the OED equations 
at large times, which shows a possible family of solutions related to the static (r.s.b.) solution. The other one is the numerical solution of these equations. This numerical solution is in fact limited to the $D=0$ version of the general random manifold problem. This is nothing but the "toy model" of a single particle in $\mathrm{N}$ dimensions, submitted to a potential which is the sum of a quadratic well and a Brownian process [27, 28, 29, 30, 20]. In the large $N$ limit, this model can be interpreted as a long range spin glass model, and we shall show that many interesting aspects of the dynamics are kept by this toy model, as is true for the statics [26, 29]. A brief account of our work has appeared recently 31.

The equilibrium Langevin dynamics (ED) of the manifolds in the large $N$ limit has been worked out by Kinzelbach and Horner in two interesting recent papers [32, 33], following the general strategy used by Sompolinsky and Zippelius [12, 13] in spin glasses. We shall basically reconsider their approach, using the OED corresponding to a finite waiting time, in the spirit of the recent work by Cugliandolo and Kurchan on the spherical spin glass with pspin interactions [17]. Technically the difference is that in the off-equilibrium dynamics the dynamical evolution starts at a time $t_{0}=0$. Therefore the correlation function $C\left(t, t^{\prime}\right)$ and the response function $r\left(t, t^{\prime}\right)$ depend explicitely on both $t$ and $t^{\prime}$. In the equilibrium dynamics the time $t_{0}$ is sent to $-\infty$, and the correlation and response become functions of the differences between $t$ and $t^{\prime}: C_{e q}\left(t-t^{\prime}\right)$ and $r_{e q}\left(t-t^{\prime}\right)$.

As we shall see there are many formal similarities between these two dynamics, together with formal similarities with the static r.s.b. solution. However one should keep in mind that the physical contents of these two approaches are actually quite different. In ED, $C_{e q}$ and $r_{e q}$ satisfy coupled equations which depend explicitely on an anomaly of the response occuring on infinite time scales. One must assume the existence of a regularization of these diverging times by considering for instance a system with a finite number of degrees of freedom. The "dynamical" equations on diverging time scales turn out to be identical to the static (r.s.b.) equations of the replica method. It is important to notice that this "dynamics" on diverging time scales is not really a dynamical solution (for instance it is invariant under arbitrary reparametrizations of time). In our opinion this equilibrium "dynamics", considered on diverging time scales, rather gives an "intuitive" and appealing description of the strange algebra of the replica method [34.

In contrast, in $\mathrm{OED}, C\left(t, t^{\prime}\right)$ and $r\left(t, t^{\prime}\right)$ obey causal equations which have a unique solution (for instance, for $t>t^{\prime}, \partial C\left(t, t^{\prime}\right) / \partial t$ depends only on $C$ and $r$ evaluated at times smaller than t.) 17. One can work directly with an infinite system, and there is no need to introduce diverging time scales. An important point is that the introduction of a finite waiting time provides a natural regularization: as we shall see, the roles of the diverging time scales are then played by some functions (e.g. powers) of the waiting time.

It is not easy to get some analytical information on the correlation and response in OED. However, as they obey causal equations, one can solve them numerically in a rather straightforward way. Our work is based on a 
detailed numerical solution of these OED equations of the toy model. We shall divide our results into two groups. One which refers to the asymptotic regime $\left(t-t^{\prime}\right.$ finite), the other refers to the non asymptotic regime.

In the asymptotic regime, we shall present hereafter numerical evidence that: 1) There exists a limiting response function $r_{a s}(\tau)=\lim _{t_{w} \rightarrow \infty} r\left(t_{w}+\right.$ $\left.\tau, t_{w}\right)$; 2) This function is the same as that derived in ED [32], with a certain condition of criticality of the anomalous response coming from diverging time scales; 3) Similar results hold for the correlation. In particular, the values of $C_{a s}(0)$ and $C_{a s}(\tau \rightarrow \infty)$ agree with the results for the statics from the full r.s.b. solution; 4) The energy $E(\tau)$ also converges to its static r.s.b. value at large $\tau$.

These results on the asymptotic behaviour provide an independent confirmation of both the static r.s.b. approach, as well as the usual equilibrium dynamics on finite time scales. In order to understand the origin of these results, and simultaneously to study the aging effects, one needs a careful study of the correlation and response for finite waiting times. Here we shall point out a few effects: 1) The very fact that one recovers the static r.s.b. results in the spin glass phase implies that there must be aging effects (in the sense that, at an arbitrary large time $t$, some perturbation of the system at times $t^{\prime}<t$ has a relevant effect, even when $t-t^{\prime}$ is very large). These aging effects are also seen in our numerical studies on the (short) time scales we can achieve. 2) It is possible to find a family of approximate solutions of dynamical equations at large times. These solutions are technically related to the solutions of the dynamics on diverging time scales found in [33], but the role of the "diverging time scales" is now played by some functions of the waiting times (like for instance $t_{w}^{u}$ ).

In the next section we introduce the model and write down the dynamical equations in the large $\mathrm{N}$ limit. In sect. 3 we review the static results obtained with the replica method. Sect. 4 presents an analytic study of the asymptotic regime, which is compared to the numerical integration of the equations

in sect. 5. Sect. 6 deals with the aging regime. Some perspectives are summarized in sect.7.

\section{The model}

The manifold is decribed by a $N$ component field $\phi_{\alpha}(x)$, where $\alpha \in 1, \ldots N$. The energy is:

$$
H=\int d^{D} x\left(\sum_{\mu=1}^{D} \sum_{\alpha=1}^{N}\left(\frac{\partial \phi_{\alpha}}{\partial x_{\mu}}\right)^{2}+\frac{\mu}{2} \sum_{\alpha=1}^{N} \phi_{\alpha}^{2}+\int d x V(x, \phi(x))\right) .
$$

where $V$ is a gaussian random potential, the correlations of which are taken as:

$$
\overline{V(x, \phi) V\left(x^{\prime}, \phi^{\prime}\right)}=-N \delta\left(x-x^{\prime}\right) f\left(\frac{\left(\phi-\phi^{\prime}\right)^{2}}{N}\right),
$$


with:

$$
f(b)=\frac{(\theta+b)^{1-\gamma}}{2(1-\gamma)}
$$

We assume a Langevin dynamics:

$$
\frac{\partial \phi_{\alpha}(x, t)}{\partial t}=-\frac{\partial H}{\partial \phi_{\alpha}(x, t)}+\eta_{\alpha}(x, t),
$$

where $\eta$ is a white noise with $<\eta_{\alpha}(x, t) \eta_{\alpha^{\prime}}\left(x^{\prime}, t^{\prime}\right)>=2 T \delta_{\alpha \alpha^{\prime}} \delta\left(x-x^{\prime}\right) \delta\left(t-t^{\prime}\right)$. This dynamics can be studied by usual field theoretical techniques [12] which are reviewed, in the present context, in [32]. We present an alternative derivation of the equations, based on the cavity method [1], in the appendix. For the OED, we find that, in the large $\mathrm{N}$ limit, the correlation:

$$
C\left(x, t ; x^{\prime}, t^{\prime}\right)=<\frac{1}{N} \sum_{\alpha} \phi_{\alpha}(x, t) \phi_{\alpha}\left(x^{\prime}, t^{\prime}\right)>
$$

and the response:

$$
r\left(x, t ; x^{\prime}, t^{\prime}\right)=<\frac{1}{N} \sum_{\alpha} \frac{\partial \phi_{\alpha}(x, t)}{\partial \eta_{\alpha}\left(x^{\prime}, t^{\prime}\right)}>
$$

satisfy the following equations: For $t>t^{\prime}$ :

$$
\begin{aligned}
\frac{\partial r\left(x, t ; x^{\prime}, t^{\prime}\right)}{\partial t}= & \left(\Delta_{x}-\mu\right) r\left(x, t ; x^{\prime}, t^{\prime}\right)+\int_{0}^{t} d s m(t, s ; x)\left(r\left(x, t ; x^{\prime}, t^{\prime}\right)-r\left(x, s ; x^{\prime}, t^{\prime}\right)\right) \\
\frac{\partial C\left(x, t ; x^{\prime}, t^{\prime}\right)}{\partial t} & =\left(\Delta_{x}-\mu\right) C\left(x, t ; x^{\prime}, t^{\prime}\right)+2 \int_{0}^{t^{\prime}} d s w(t, s ; x) r\left(x, t^{\prime} ; x^{\prime}, s\right) \\
& +\int_{0}^{t} d s m(t, s ; x)\left(C\left(x, t ; x^{\prime}, t^{\prime}\right)-C\left(x, s ; x^{\prime}, t^{\prime}\right)\right)
\end{aligned}
$$

and:

$$
\begin{aligned}
\frac{1}{2} \frac{d C\left(x, t ; x^{\prime}, t\right)}{d t} & =\left(\Delta_{x}-\mu\right) C\left(x, t ; x^{\prime}, t\right)+2 \int_{0}^{t} d s w(t, s ; x) r\left(x, t^{\prime} ; x^{\prime}, s\right) \\
& +\int_{0}^{t} d s m(t, s ; x)\left(C\left(x, t ; x^{\prime}, t\right)-C\left(x, s ; x^{\prime}, t\right)\right)+T .(9)
\end{aligned}
$$

In these equations, we have used the following notations:

$$
\begin{aligned}
w\left(t, t^{\prime} ; x\right) & =f^{\prime}\left(b\left(t, t^{\prime} ; x\right)\right), m\left(t, t^{\prime}, x\right)=4 f^{\prime \prime}\left(b\left(t, t^{\prime} ; x\right)\right) r\left(x, t ; x, t^{\prime}\right) \\
b\left(t, t^{\prime} ; x\right) & =C(x, t ; x, t)+C\left(x, t^{\prime} ; x, t^{\prime}\right)-2 C\left(x, t ; x, t^{\prime}\right) .
\end{aligned}
$$

This set of equation is causal. The boundary conditions on $r$ are $r\left(x, t, x^{\prime}, t^{-}\right)=$ $\delta\left(x-x^{\prime}\right)$. Given an initial condition $C\left(x, 0, x^{\prime}, 0\right)$, it has a unique solution.

In the following we shall concentrate on the toy model, $D=0$, where the space dependence in these equations is dropped. We note that in this limit the model, described by the simple Hamiltonian $H=(1 / 2) \mu \sum_{\alpha} \phi_{\alpha}^{2}+$ $V\left(\phi_{1}, \ldots, \phi_{N}\right)$, admits another interesting interpretation as a spin-glass. The components $\phi_{a}$ can be thought as soft spins in a quadratic well, interacting via the random potential $V$. In particular, in its spherical version, i.e. taking the constraint $\frac{1}{N} \sum_{\alpha} \phi_{\alpha}^{2}=1$, it is possible to choose the values of $\theta$ and $\gamma$ such as to obtain the spherical $p$-spin model considered in [35, 17]. 


\section{Static replica solution}

We briefly review here the results of the static r.s.b. approach for the toymodel $(D=0)$, concentrating on quantities that we will study in dynamics. We keep to the case of "long range" disorder correlation $\gamma<1$ where the replica symmetry breaking is of the full continuous kind. The equilibrium statistical mechanics of the model has been studied in [26, 29] for the special case $\theta=0$. In dynamics a non-zero $\theta$ is needed to regularize the correlations of the potential at short distance. The results of [26, 29] generalize as follows. At high temperature the system is ergodic and replica symmetric, and the equilibrium is characterized by the correlations

$$
\begin{gathered}
\frac{1}{N} \sum_{\alpha}{\overline{\left\langle\phi_{\alpha}^{2}\right\rangle_{\text {Gibbs }}}}_{\alpha}=\tilde{q} \frac{T}{\mu}+\frac{1}{\mu^{2}}\left(\theta+\frac{2 T}{\mu}\right)^{-\gamma} \\
\frac{1}{N} \sum_{\alpha}{\overline{\left\langle\phi_{\alpha}\right\rangle^{2}}}_{\text {Gibbs }}=q=\frac{1}{\mu^{2}}\left(\theta+\frac{2 T}{\mu}\right)^{-\gamma}
\end{gathered}
$$

where by angular brackets we have denoted the thermal average and by an overline the disorder average. The energy is given by

$$
E=\frac{\mu}{2} \tilde{q}+\frac{1}{T}[f(0)-f(2(\tilde{q}-q))]
$$

At a critical temperature $T_{c}$,

$$
T_{c}=\frac{\mu}{2}\left(-\theta+\frac{\mu^{2}}{2 \gamma}\right)^{\left(\frac{-1}{1+\gamma}\right)}
$$

there is a phase transition and replica symmetry is broken. The thermodynamics of the system is fully specified by $\tilde{q}=\frac{1}{N} \sum_{\alpha} \overline{\left\langle\phi_{a}^{2}\right\rangle}$ and by a function $q(u), u \in[0,1]$. Standard arguments from the mean field theory of spin glasses [1], imply breaking of ergodicity and the existence of many pure states, whose correlations are characterized by a non trivial $P(q)$ defined as the overlap distribution for two copies of the system with identical realization of the random potential $V$ :

$$
P(q)=\overline{<\frac{1}{N} \sum_{\alpha=1}^{N} \delta\left(\phi_{\alpha} \psi_{\alpha}-q\right)>_{G i b b s}}=\frac{\mathrm{d} u(q)}{\mathrm{d} q}
$$

where $u(q)$ is the inverse function of $q(u)$. The order parameter function $q(u)$ is:

$$
q(u)= \begin{cases}q_{0} & u \leq u_{0} \\ \tilde{q}+\frac{\theta}{2}-\frac{1}{2}\left(\frac{\sqrt{2 \gamma}}{1+\gamma} \frac{u}{T}\right)^{2 /(\gamma-1)} & u_{0} \leq u \leq u_{1} \\ q_{1} & u_{1} \leq u \leq 1\end{cases}
$$

where

$$
q_{0}=\frac{1}{2 \gamma}\left(\frac{\mu^{2}}{2 \gamma}\right)^{-1 /(1+\gamma)}
$$




$$
\begin{aligned}
u_{0} & =T(1+\gamma)(\mu)^{(1-\gamma) /(1+\gamma)}(2 \gamma)^{-1 /(1+\gamma)} \\
u_{1} & =\frac{T}{\sqrt{2 \gamma}}(1+\gamma)\left(\theta+2\left(\tilde{q}-q_{1}\right)\right)^{(\gamma-1) / 2} \\
\tilde{q} & =\frac{1+\gamma}{2 \gamma}\left(\frac{\mu^{2}}{2 \gamma}\right)^{-1 /(1+\gamma)}-\theta / 2
\end{aligned}
$$

and $\tilde{q}-q_{1}$ is the solution of the equation

$$
\tilde{q}-q_{1}=\frac{T}{\sqrt{2 \gamma}}\left(\theta+2\left(\tilde{q}-q_{1}\right)\right)^{(1+\gamma) / 2} .
$$

From the knowledge of $\tilde{q}$ and $q(u)$ all the physical quantities at equilibrium can be calculated, for example the energy is:

$$
E=\frac{\mu}{2} \tilde{q}+\frac{1}{T}\left[f(0)-\int_{0}^{1} \mathrm{~d} u f(2(\tilde{q}-q(u)))\right]
$$

The results presented here for the $q(u)$ have also been obtained in 33 in the ED approach with Sompolinsky ansatz, which, as we have already remarked, reproduces the algebra of the r.s.b. approach.

\section{Analytic study of the asymptotic regime}

The scope of this section is to study the behaviour of the solution of the dynamical equations $(7,8,9,9)$ in the "asymptotic" limit. This limit is defined as $t=t_{w}+\tau, t^{\prime}=t_{w}$, with $t_{w} \rightarrow \infty$ while $\tau$ is kept fixed. For the sake of the simplicity of the presentation, we shall present the whole analysis in the case $D=0$. The generalization of the analytic results to higher dimensional problems is straightforward. We rewrite here, just for graphical transparency, the dynamical equations $(7,8,9,9)$ for $D=0$.

$$
\begin{aligned}
\frac{\partial r\left(t, t^{\prime}\right)}{\partial t} & =-\mu r\left(t, t^{\prime}\right)+\int_{0}^{t} d s m(t, s)\left(r\left(t, t^{\prime}\right)-r\left(s, t^{\prime}\right)\right) \\
\frac{\partial C\left(t, t^{\prime}\right)}{\partial t} & =-\mu C\left(t, t^{\prime}\right)+2 \int_{0}^{t^{\prime}} d s w(t, s) r\left(t^{\prime}, s\right) \\
& +\int_{0}^{t} d s m(t, s)\left(C\left(t, t^{\prime}\right)-C\left(s, t^{\prime}\right)\right) \\
\frac{1}{2} \frac{d C(t, t)}{d t} & =-\mu C(t, t)+2 \int_{0}^{t} d s w(t, s) r(t, s) \\
& +\int_{0}^{t} d s m(t, s)(C(t, t)-C(s, t))+T
\end{aligned}
$$

with

$$
\begin{aligned}
w\left(t, t^{\prime}\right) & =f^{\prime}\left(b\left(t, t^{\prime}\right)\right), m\left(t, t^{\prime}\right)=4 f^{\prime \prime}\left(b\left(t, t^{\prime}\right)\right) r\left(t, t^{\prime}\right) \\
b\left(t, t^{\prime}\right) & =C(t, t)+C\left(t^{\prime}, t^{\prime}\right)-2 C\left(t, t^{\prime}\right) .
\end{aligned}
$$


For future reference we also give the formula for the energy:

$$
E(t)=\frac{\mu}{2} C(t, t)-2 \int_{0}^{t} \mathrm{~d} s f^{\prime}(b(t, s)) r(t, s)
$$

Let us make the reasonable assumption, supported by the numerical integration below, of the existence of an asymptotic regime for $t, t^{\prime} \rightarrow \infty$ keeping $\tau=t-t^{\prime}$ finite. Namely we will suppose the existence of the two limiting functions

$$
\begin{aligned}
r_{a s}(\tau) & =\lim _{t^{\prime} \rightarrow \infty} r\left(t^{\prime}+\tau, t^{\prime}\right) \\
C_{a s}(\tau) & =\lim _{t^{\prime} \rightarrow \infty} C\left(t^{\prime}+\tau, t^{\prime}\right) .
\end{aligned}
$$

Taking the limit of the dynamical equations (19) in the asymptotic regime, we get the non causal equations

$$
\begin{aligned}
\frac{d b_{a s}}{d \tau} & =\left(-\mu+M_{a s}+\bar{M}\right) b_{a s}(\tau)-\int_{0}^{\tau} d \tau^{\prime} m_{a s}\left(\tau-\tau^{\prime}\right) b_{a s}\left(\tau^{\prime}\right)+2 T \\
& -\int_{0}^{\infty} d \tau^{\prime}\left[m_{a s}\left(\tau+\tau^{\prime}\right)-m_{a s}\left(\tau^{\prime}\right)\right] b_{a s}\left(\tau^{\prime}\right)+4\left[w_{a s}\left(\tau+\tau^{\prime}\right)-w_{a s}\left(\tau^{\prime}\right)\right] r_{a s}\left(\tau^{\prime}\right), \\
\frac{d r_{a s}}{d \tau} & =\left(-\mu+M_{a s}+\bar{M}\right) r_{a s}(\tau)-\int_{0}^{\tau} d \tau^{\prime} m_{a s}\left(\tau-\tau^{\prime}\right) r_{a s}\left(\tau^{\prime}\right), \\
C_{a s}(0) & =\frac{1}{\mu-\bar{M}}\left(T+\frac{1}{2} \int_{0}^{\infty} d s m_{a s}(s) b_{a s}(s)+2 \int_{0}^{\infty} d s w_{a s}(s) r_{a s}(s)\right)
\end{aligned}
$$

where for convenience we have written the equation for the correlation in terms of $b_{a s}(\tau)=2\left[C_{a s}(0)-C_{a s}(\tau)\right]$ instead of $C_{a s}(\tau)$ and we have denoted

$$
\begin{gathered}
M_{a s} \equiv \int_{0}^{\infty} \mathrm{d} \tau m_{a s}(\tau), \\
\bar{M} \equiv \lim _{t \rightarrow \infty} \int_{0}^{t} \mathrm{~d} s m(t, s)-M_{a s} .
\end{gathered}
$$

The functions $m_{a s}$ and $w_{a s}$ are defined in a way similar to $m$ and $w$ in (20), but using the asymptotic correlation and response.

The term $\bar{M}$, which we will call "anomaly" in the following, is the term which couples the asymptotic time regime $\left(\tau=t-t^{\prime}\right.$ finite) to the non asymptotic ones. The equations $(25)$ are identical to those which appear in the ED studied by Kinzelbach and Horner 33. The only difference lies in the interpretation of the anomaly: In ED it is supposed to be due to the response of the system to some perturbations taking place on infinite time scales. This is not easy to define, since the regularization of these diverging time scales by using a finite volume system in principle invalidates the derivation of the dynamical equations (25). The definition (26) of the anomaly in OED is very clear.

Let us now briefly quote the following results from the study of ED in [33]: One may search a solution of the asymptotic equations (25) which satisfies the fluctuation-dissipation-theorem (f.d.t.):

$$
\operatorname{Tr}_{a s}(\tau)=-\frac{\partial}{\partial \tau} C_{a s}(\tau)=\frac{1}{2} \frac{\partial}{\partial \tau} b_{a s}(\tau) .
$$


Assuming the f.d.t., the asymptotic equations simplify to:

$$
\frac{d b_{a s}}{d \tau}=2 T-b_{a s}(\tau)\left(\mu-\bar{M}-\int_{\tau}^{\infty} d s m_{a s}(s)\right)+\int_{0}^{\tau} d s m_{a s}(s)\left(b_{a s}(\tau)-b_{a s}(s)\right)
$$

The condition for the existence of a monotonous solution $b(t)$ to this equation is that

$$
b_{a s}(\infty)=\frac{2 T}{\mu-\bar{M}}<b_{m},
$$

where $b_{m}$ is the point where the function of $b: T / b-f^{\prime}(b) / T$ is minimal. There are two regimes: at temperatures above the critical temperature $T_{c}$ which equals the value (13) computed within the static approach, there exists a solution when the anomaly $\bar{M}$ is zero. This solution agrees with the static replica symmetric results (11):

$$
b_{a s}(\infty)=\frac{2 T}{\mu}=2(\tilde{q}-q), C_{a s}(0)=\tilde{q},
$$

At low temperatures, $T<T_{c}$, there is no solution satisfying the f.d.t. relation if $\bar{M}=0$. For such a solution to exist one needs a non zero anomaly: $\bar{M} \leq \mu-2 T / b_{m}<0$. The special choice (named "postulate of marginal stability" in [33]) of the anomaly:

$$
\bar{M}=\mu-2 T / b_{m}
$$

leads to an asymptotic correlation $b_{a s}(\infty)=b_{m}$, which is equal to the static result: $2\left(\tilde{q}-q_{1}\right)$ computed within the static approach with r.s.b. (17). Similarly, one gets $C_{a s}(0)=\tilde{q}$

We can summarize this discussion about the asymptotic dynamics in the low temperature phase as follows: In view of the static analysis, and its interpretation in terms of ergodicity breaking, it is reasonable to assume the existence of an asymptotic regime, obeying the f.d.t., and such that the two following static correlations are recovered: $C_{a s}(0)=\tilde{q}, C_{a s}(\infty)=\tilde{q}-q_{1}$. However for such a regime to exist one needs a non zero value of the anomaly. In the next section we present some numerical results which confirm the validity of these assumptions, in section 6 we study the implications of the existence of an anomaly in terms of aging.

\section{Numerical study of the asymptotic regime}

While the set of assumptions which have been put forward at the end of the previous section look very reasonable, they still deserve a confirmation. (In fact some models have been found, such as the spherical spin glass with $\mathrm{p}(\geq 3)$ spin interactions, where even the values of the critical temperatures found in the static and dynamic approaches are different [36, 17, 35]. It is believed that this effect is related to the fact that the replica symmetry

breaking is first order in these models.) If these assumptions are correct, it 
means that the system of causal first order equations (77, 8) contains the static solution with full replica symmetry breaking, which is in itself an interesting observation.

In this section we present a numerical study of the dynamical equations (19). Our aim is to study the low temperature phase of the model comparing the result of the integration with the static solution and the asymptotics of the previous section. The values of the parameters appearing in the Hamiltonian have been chosen equal to $\gamma=1 / 2, \mu=1 / 8, \theta=5$. With this choice the critical temperature is $T_{c}=.658$, and for $T \leq T_{c}$ the static correlations take the value $\tilde{q}=21.5$

The discretization of (19) was chosen to be the simple one induced by the discretization of the Langevin equation (4) with the Ito convention. We have solved the discrete equations with time steps $4 h, 2 h$, and $h$, and extrapolated the correlation and response to $h=0$ by a second degree polynomial. $h$ was chosen in such a way that this extrapolation does not differ too much from the linear extrapolation of the data at $2 h$ and $h$. In this way with $h=.3$ we where able to reach times of the order of 1000 . We also performed the integration of the equation for longer times for some particular value of $h$, as we will specify in the following. In most of the simulation the initial condition $C(0,0)=21.5$ was taken. We have checked that the dynamics in the asymptotic region does not depend on this choice.

We have integrated the system (19) for $T=.5$ and $T=.2$. A run was also performed at $T=3>T_{c}$. With this last run we checked that in the high temperature phase the OED simply corresponds to the relaxation into the unique equilibrium state described by the r.s. statics. Coherently we find that $C(t, t)$ tends exponentially to its r.s. value $\tilde{q}_{r s}=32.8$, and the energy to $E_{r s}=0.368$. In the low temperature phase the situation changes. The asymptotic extrapolation for $C(t, t)$ and $E(t)$ become incompatible with the r.s. values. As a first approximation, the behaviour of the equal time correlations $C(t, t)$ is compatible with a power law approach to its asymptotic value (with an exponent, deduced from the behaviour of $d C(t, t) / d t$, equal to $-.73 \pm .05)$ 31]. When one uses this power law fit in order to extrapolate $C(t, t)$ to infinite $t$, it yields the result $21.4 \pm .1$ which is in agreement with the r.s.b. prediction 21.5. For lower temperatures this procedure is less precise, and there are clearly corrections to the simple power law behaviour of $d C(t, t) / d t$. Better estimates for the asymptote are obtained fitting the time derivatives of $C(t, t)$ and $E(t)$ with functions depending on three parameters:

$$
\begin{aligned}
& f_{1}(t)=a_{1} t^{-a_{2}}\left(1+\frac{a_{3}}{t}\right) \\
& f_{2}(t)=a_{1} t^{-a_{2}}(\log (t))^{a_{3}}\left(1-a_{2}+\frac{a_{3}}{\log (t)}\right) .
\end{aligned}
$$

In the time window we reach, these two fits give comparable errors, but also comparable estimates for the asymptote (after integration of the fits). For instance we show in Fig. 1a an estimate of the large time limit, $C_{\infty}$, of the autocorrelation $\mathrm{C}(\mathrm{t}, \mathrm{t})$, from the numerical solution of the dynamical equations 
with a grid size $h=1.2$. The derivative $d C(t, t) / d t$ has been fitted to the function $f_{2}$. For each time $t, C_{\infty}$ is approximated by $C(t, t)$ plus the integral of the fit of the derivative. The plot gives $C(t, t)+\int_{t}^{\infty} f_{2}\left(t^{\prime}\right) d t^{\prime}$ versus the time $t$. Fig. $1 \mathrm{~b}$ shows that the effect of the interpolation at $h=0$ become small at large time. Altogether this procedure gives $C_{\infty} \simeq 21.49$, with an error, due to the fit, the extrapolations, which we estimate subjectively to \pm .05 . This is quite compatible with the analytical result from r.s.b., $C_{\infty}=\tilde{q}=21.5$. In Fig.2 we give the analogous plots for the energy. The correction due to the finite grid size do not vanish at long times and must be incorporated. We get as a final result: $E_{\infty}=-1.366 \pm .02$, in very good agreement with the r.s.b. computation: $E_{\infty}=-1.3660$. Similar results can be found at a temperature $T=.2$. Probably the best evidence for the convergence of $C(t, t)$ to $\tilde{q}$ is obtained considering the quantity

$$
A(t)=\tilde{q}(1-r(t, 0))-C(t, t) .
$$

and observing that $r(t, 0)$, the response at time $t$ to a change in the field at time zero, should tend to zero at large time. So if $C(t, t)$ converges to $\tilde{q}, A(t)$ must go to 0 at large times. In Fig. $3 \mathrm{~A}(\mathrm{t})$, as well as $B(t)=\tilde{q}-C(t, t)$ are plotted on a log-log scale for $\mathrm{T}=.2$. A pure power law fit gives:

$$
\begin{aligned}
& A(\infty)=.04 \\
& B(\infty)=.3,
\end{aligned}
$$

the quality of this two parameter fit on $A$ is comparable with the ones we had on $C$ with logarithmic or power law corrections.

Let us now turn to the study of the asymptotic functions $b_{a s}$ and $r_{a s}$. In Fig. 4a we plot for $T=.5$ the response $r\left(t_{w}+\tau, t_{w}\right)$ versus $\tau$ for various values of the waiting time $t_{w}$. We also give the result $r_{a s}(\tau)$ of a 3 parameter power law extrapolation of these data at $t_{w}=\infty$. The same is done in Fig. $4 \mathrm{~b}$ for the correlation $b\left(t_{w}+\tau, t_{w}\right)=C\left(t_{w}+\tau, t_{w}+\tau\right)+C\left(t_{w}, t_{w}\right)-2 C\left(t_{w}+\right.$ $\left.\tau, t_{w}\right)$. According to the statics, the correlation should go to $\lim _{\tau \rightarrow \infty} b_{a s}(\tau)=$ $2\left(\tilde{q}-q_{1}\right)=6.068$. It is possible to see directly that the data is compatible with this asymptota, with a power law approach. However, in view of the relatively short times $\tau$ accessible here (keeping $\tau<<t_{w}$ ), we prefer to use a different approach which is the comparison to an analytic study of the asymptotic equations. In Fig.4 the limiting functions obtained from a power law interpolation are compared to those obtained from the numerical integration of the asymptotic equations (25) with the anomaly set to its "marginal" value (31). The agreement is very good. This confirms that the asymptotic dynamics coincides with the ED on finite timescales, and agrees with the static r.s.b. results.

\section{The non asymptotic regime: aging}

We now turn to the non asymptotic times. From the previous sections we know that there exists a non zero "anomaly". This means that the decay 
of the response $r(t, s)$ at large $t-s$ is slow. More precisely, it implies that the integrated response at a large time $t, \int_{0}^{t} r(t, t-\tau) \mathrm{d} \tau$, receives some finite contributions from time differences $\tau$ which diverge when $t$ goes to infinity. We define such a situation as a situation of aging. This definition is compatible with the ones used so far. It basically means that even at large times the physics of the system depends on its previous history. Besides the usual asymptotic regime $t \rightarrow \infty, t^{\prime} \rightarrow \infty, t-t^{\prime}$ finite, there exist other "crossover regimes", in which the limit $t, t^{\prime} \rightarrow \infty$ is taken in a different way. The asymptotic regime cannot be decoupled from these other regimes.

We now propose a solution of the dynamical equations, giving the correct result for the anomaly, in the non asymptotic regime. Basically we propose a reformulation of the Sompolinsky Ansatz [13, 33] in the context of OED. The main difference is that here we do not impose temporal homogeinity in the equations $a b$ initio. The diverging time scales of Sompolinsky's approach, needed for the system to cross the diverging barriers, are here substituted by some function of the waiting time $t_{w}$, which provides a natural cut-off for the theory. A simple version of this scenario, including one single crossover domain (corresponding to a single step of r.s.b.), had been found by Cugliandolo and Kurchan in the spherical p-spin model [17]. Recently they have also proposed a similar scenario for the OED of the Sherrington Kirpatrick model close to its critical temperature [37]. Let us perform the limit $t, t^{\prime} \rightarrow \infty$ by dividing the octant $t^{\prime} \leq t$ into non-overlapping crossover domains. A crossover domain $\mathcal{D}_{u}$ is defined, using an increasing function $h_{u}(t)$, as the set of times $t, t^{\prime}$ which are both large, but keeping the ratio $h_{u}\left(t^{\prime}\right) / h_{u}(t)=\exp (-\tau)$ fixed, with $\tau \in] 0, \infty\left[\right.$. . Suppose that in the crossover domain $\mathcal{D}_{u}$ one has:

$$
b\left(t, t^{\prime}\right)=\hat{b}_{u}(\tau), r\left(t, t^{\prime}\right)=\frac{d \ln \left[h_{u}\left(t^{\prime}\right)\right]}{d t^{\prime}} \hat{r}_{u}(\tau) .
$$

Then the contribution to the anomaly $\int_{0}^{t} d s m(t, s)$ from all the times $s$ such that $s$ and $t$ are in $\mathcal{D}_{u}$ is finite and equal to

$$
\int_{0}^{\infty} \mathrm{d} \tau 4 f^{\prime \prime}\left(\hat{b}_{u}(\tau)\right) \hat{r}_{u}(\tau)
$$

which is independent on the function $h_{u}$.

In a simple problem like for instance the high temperature phase, there should exist a single crossover domain, the asymptotic one defined by $h(t)=$ $e^{t}$. In a glass phase, we can have a relatively simple scenario in which there exists, beside the asymptotic domain, another one defined by some other function $h(t)$. Such a case (with $h(t)=t$ ) has been found recently [17]. But one can also have some systems with many crossover domains. The condition we impose is that they do not overlap. We can index them by a

${ }^{2}$ The index $u$ of the domains should at first be taken as a discrete variable, in a procedure analogous to that of statics in which one considers first a finite number of r.s.b. and then passes to the continuum limit. This is familiar to the reader both from the static r.s.b. approach and from the ED, and it will be not repeted here. We just mention that $u$ will turn out to be a continuous variable in the interval $[0,1]$. 
parameter $u$ such that, if $w<u<v$ and the points $\left(t, t^{\prime}\right)$ belong to $\mathcal{D}_{u}$, then $h_{v}\left(t^{\prime}\right) / h_{v}(t)=0$ and $h_{w}\left(t^{\prime}\right) / h_{w}(t)=1$. A possible choice leading to such a behaviour would be for instance $h_{u}(t)=\exp \left(t^{u}\right)$. With this choice the points $\left(t, t^{\prime}\right)$ belong to $\mathcal{D}_{u}$ when $t^{\prime}=t-t^{(1-u)} \tau / u$.

The alert reader will have recognised in this scenario a hierarchical stucture which is reminiscent of the ultrametricity assumption underlying both the statics and the equilibrium dynamics [38]. We have here a hierarchy of time crossovers. Considering three times $t^{\prime \prime}<t^{\prime}<t$, one sees that, if $\left(t, t^{\prime}\right)$ belongs to the crossover domain $\mathcal{D}_{u}$ and $\left(t^{\prime}, t^{\prime \prime}\right)$ belongs to $\mathcal{D}_{v}$, then $\left(t, t^{\prime \prime}\right)$ belongs to $\mathcal{D}_{\text {inf }(u, v)}$, which is an ultrametric inequality, and obviously implies ultrametric relations for the corresponding correlation functions.

The dynamical equations can be solved within this scenario because one can forget the time derivatives in the dynamical equations. The existence of an asymptotic regime in which $\lim _{\tau \rightarrow \infty} \partial C_{a s}(\tau) / \partial \tau=0$, implies that in the crossover regimes $\frac{\partial C\left(t, t^{\prime}\right)}{\partial t^{\prime}} \rightarrow 0$ while $\frac{\partial r\left(t, t^{\prime}\right)}{\partial t^{\prime}}$ tends to zero more rapidly then $r\left(t, t^{\prime}\right)$. The 1.h.s. of (19) can be neglected in this situation and the problem becomes invariant under the family of transformations

$$
\begin{aligned}
C\left(t, t^{\prime}\right) & \rightarrow C\left(h(t), h\left(t^{\prime}\right)\right) \\
r\left(t, t^{\prime}\right) & \rightarrow \frac{\mathrm{d} h\left(t^{\prime}\right)}{\mathrm{d} t^{\prime}} r\left(h(t), h\left(t^{\prime}\right)\right)
\end{aligned}
$$

for any monotonically increasing function of time $h(t)$. Any non trivial solution will break this invariance, consequently from a given solution we can generate a whole "orbit" of equivalent ones just reparametrizing the time. As we have already remarked, the solution of (19) is unique at any finite times $t, t^{\prime}$. The appearence of this invariance seems somewhat artificial; among all these possible solutions, only one can be the asymptote of the finite time dynamics. At this stage it is an open problem what is the choice which will be picked up by the dynamics.

The ambiguity due to the time reparametrization invariance of the asymptotic equations reflects in the fact that the equations for $\hat{b}_{u}$ and $\hat{r}_{u}$ are independent of the choice of all the arbitrary functions $h_{u}(t)$. In fact these equations are identical to those derived in ED on diverging time scales; this set of equations has been shown [33] to possess solutions satisfying the "quasi f.d.t." relation:

$$
u \frac{\mathrm{d} \hat{b}_{u}}{\mathrm{~d} \tau}=2 T \hat{r}_{u}(\tau)
$$

Denoting $b_{u}^{+}=\hat{b}_{u}(0)$ and $b_{u}^{-}=\hat{b}_{u}(\infty)$ one has for adjacent domains indexed by $u<u^{\prime}, b_{u}^{+}=b_{u^{\prime}}^{-}$. Within the OED, we find that the dynamical correlations are related to the static order parameter function $q(u)$ by the formula

$$
b_{u}^{-}=2(\tilde{q}-q(u))
$$

With these ingredients we reproduce the algebra of the static replica solution, which gives the value (31) for the anomaly. In each domain, apart from the 
asymptotic one, the variation with $\tau$ of the functions $\hat{b}_{u}(\tau)$ is infinitesimal, and $q(u)$ becomes the continuous function given by (15).

As we stressed before, this solution can be understood as a reinterpretation of the ED solution, and of the static r.s.b. solution. With respect to the ED solution, the main advantage is that the diverging time scales have been replaced basically by some powers of the waiting time. Unfortunately it does not solve the second problem of ED, namely the invariance through reparametrizations of time which implies that one looses all the physical (crossover) time scales. We stress that this is only a problem of the family of solutions that we have introduced. This problem is not intrinsic to the OED itself. On the contrary, in the real OED problem there is a unique solution to the dynamical equations. This solution might go asymptotically to one of the solutions we have presented here (choosing dynamically a set of functions $h_{u}(t)$ ), or it might even converge to some other asymptote. So far we have not been able to answer this problem analytically. So we shall now propose some numerical checks which proceed through the numerical solution of the dynamical equations.

The numerical test of this family of solutions might seem hopeless insofar as they depend on an arbitrary set of functions $h_{u}(t)$ which allow for a reparametrization of time. We shall call such a set a choice of gauge. In order to decide whether the asymptotic solution belongs to our family, we propose to use criteria which are gauge independent. One possibility is to use some integrated quantities like the "dynamical moments" introduced in [17]:

$$
\mathcal{C}_{k}(t) \equiv k \int_{0}^{t} d s \operatorname{Tr}(t, s) C(t, s)^{k-1},
$$

Within our scenario of hierarchical crossover domains, these moments should have a large time limit given by:

$$
\lim _{t \rightarrow \infty} \mathcal{C}_{k}(t)=\tilde{q}^{k}-\int \mathrm{d} q P(q) q^{k} .
$$

Another possibility consists in the introduction of the function:

$$
U\left(t, t^{\prime}\right)=\frac{\operatorname{Tr}\left(t, t^{\prime}\right)}{\frac{\partial C\left(t, t^{\prime}\right)}{\partial t^{\prime}}}
$$

In the crossover regime, where the f.d.t. relation holds, $U$ takes the value $U\left(t, t^{\prime}\right) \equiv 1$ at large times, while, in the crossover domain it gives us a measure of the violation of f.d.t.. We shall call this function the fluctuation dissipation (f.d.) ratio. The gauge invariant prediction of the hierarchical crossover domains scenario is that, if one plots the f.d. ratio $U$ as a function of the time $t$ along the lines of fixed correlation $C$, its value at large times is equal to $u(q)$, the inverse of the order parameter function. Let us make this statement more precise: we first observe that for fixed (and large enough) $t$, $C\left(t, t^{\prime}\right)$ is a monotonously increasing function of $t^{\prime}$. This allows to define the function $t^{\prime}(q, t)$ as the time $t^{\prime}$ such that $C\left(t, t^{\prime}\right)=q$. The prediction is that:

$$
U_{d}(q) \equiv \lim _{t \rightarrow \infty} U\left(t, t^{\prime}(q, t)\right)=u(q)=\int_{0}^{q} d q^{\prime} P\left(q^{\prime}\right)
$$


which is the inverse of the order parameter function defined in (15). We have been able to obtain the following general results on the f.d ratio. It is easy to show that $C(t, 0)=r(t, 0) C(0,0)$. Under the reasonable assumption $\lim _{t \rightarrow \infty} r(t, 0)=0$, one gets that $\lim _{t \rightarrow \infty} C(t, 0)=0$, and it is easy to deduce that $\lim _{t \rightarrow \infty} U\left(t, t^{\prime}=0\right)=0$. We have seen numerically, but we have not been able to prove, that for large enough $t, U\left(t, t^{\prime}\right)$ is an increasing function of $t^{\prime}$. Together with the f.d.t. result in the asymptotic regime $\lim _{t \rightarrow \infty} U(t, t)=1$, this shows that $U$ tends to a probability at large times.

We have tried to use the simple dicretization algorithm described in the previous section to study these aging effects (with the same values of $h$ ). Although we shall see that the times we have reached do not allow to draw definitive conclusion on the crossover regimes, we think it is worth to present some of the data, in order to see what happens on relatively short times, and to give an idea of the type of computing effort which will be needed in order to solve this problem. The values of the parameters are $\gamma=.5, \theta=$ $5 ., \mu=.125, T=.5, C(0,0)=0$. We have checked that the errors due to the discretisation and interpolation procedures are negligible on the scales of the figures.

We first present some confirmation of the existence of the aging effect. In Fig.5 we plot the "thermoremanent magnetization" which we define as:

$$
\mathcal{M}\left(\tau, t_{w}\right)=\int_{O}^{t_{w}} d s r\left(t_{w}+\tau, s\right)
$$

The plot shows $\mathcal{M}\left(\tau, t_{w}\right)$ versus $\tau$ for fixed values of the waiting time $t_{w}$, on logarithmic scales. On these time scales, one clearly sees an aging effect which is qualitatively similar to the one observed in experiments [4, 5, 6, 7] and numerical simulations [39, 18, 19] in spin glasses. The effect is confirmed in Fig. 6 which plots the normalised correlation $C\left(t_{w}+\tau, t_{w}\right) / C\left(t_{w}, t_{w}\right)$ versus $\tau$, at fixed $t_{w}$. We have observed that the curves do not scale very well as functions of $\tau / t_{w}$.

We have tried to test the hierarchical solution by some studies of gauge invariant quantities. We first study the dynamical moments (40). The first moment $\mathcal{C}_{1}$ satisfies a kind of Ward identity (related to the translational invariance of the distribution of the random potential):

$$
r(t, 0)=1-\frac{\mu}{T} \mathcal{C}_{1}(t)
$$

(a simple proof consists in showing that the two sides of this equality satisfy the same first order differential equation in time, with the same initial condition). As $r(t, 0)$ should vanish at large times, this implies that $\lim _{t \rightarrow \infty} \mathcal{C}_{1}(t)=T / \mu=\tilde{q}-\int d q P(q) q$. Numerically we have checked that the Ward identity (45) is satisfied with a precision of $10^{-5}$, and that the behaviour of $r(t, 0)$ is consistent with a decay to zero. We have computed numerically the first five moments $\mathcal{C}_{k}(t), k=1, \ldots, 5$. In Fig.7 we plot the third moment versus time. Within the hierarchical scenario one would expect that its large time limit should be given by the third moment of the static $P(q)$ as in (41), 
which is equal to 9011 in our case. The inset of Fig.7 shows that the relative difference of $\mathcal{C}_{3}(t)$ with this value decays approximately as a power law. However at $t \simeq 800$ the relative difference is still of order 10 per cent. Fig.8 shows the fifth moment and its approach to the static value $5.7610^{6}$. We consider this data as compatible with the hierarchical scenario but not really conclusive. As explained above, a more detailed analysis of the data consists in studying the f.d. ratio (42) and to test the prediction (43). In Fig. 9 we plot $C\left(t, t^{\prime}\right) / C(t, t)$ versus the time $t$, along lines in the $t^{\prime}, t$ plane such that $U\left(t, t^{\prime}\right)$ is constant, equal to $u_{0}$. According to the hierarchical scenario, this quantity should go at large $t$ to $q\left(u_{0}\right) / \tilde{q}$ defined in (15). On this time scale, we do not see evidence for such a convergence. To summarize, we consider the results on the moments as encouraging, but the detailed analysis on the f.d. ratio shows that simulations on much longer time scales are needed in order to decide on the correctness of the hierarchical solution.

\section{Conclusions}

In this paper we have studied the off equilibrium dynamics of a disordered model which represents on one hand a limiting case of a manifold in a random environment, on the other hand a spin glass with long range interactions. The choice of this model has several motivations. Its static solution at low temperatures implies a full continuous r.s.b., as for instance in the SK model; this r.s.b. solution is known in all details. On the other hand, we can write a closed set of coupled dynamical equations between the correlation and response. Because of this, we have been able to generalize the analytic solution of 17 in the aging regime of the spherical p-spin model to a full r.s.b. case and to compare to a numerical integration of the equations. Simultaneously to our work, Cugiandolo and Kurchan have also extended their analytic solution to the SK model close to $T_{c}$ [37].

Our analysis is consistent with the existence of two regimes at large times in the low temperature phase: an asymptotic regime where time homogeneity and fluctuation dissipation relations hold, and an aging regime where both these properties are violated. These regimes are similar to the ones observed in experiments and simulations. We have found convincing numerical evidence that the asymptotic regime agrees with the static r.s.b. results and with the ED results. The correlations are those characteristic of a system reaching equilibrium inside one single valley. We have shown that these facts imply the existence of a non trivial aging regime.

We have proposed a family of solutions of the dynamics at large time in this aging regime, based on a hierarchy of crossover domains. This solutions solve the problem of the diverging time scales which had to be introduced in ED. On the other hand several problems are left open. We have not been able to show that the dynamics converges to one of these solutions, and a fortiori we do not know which of them is picked up. This choice might well depend on the choice of the Langevin dynamics and of the type of initial conditions 
which are used. We have found that the f.d. ratio tends to a probability law at large times. Longer simulations are needed to decide whether this probability law is identical to the static $u(q)$, as implied by the hierarchical scenario. At the present stage, we believe that it is crucial to carry out this numerical study. The physical interpretation of the dynamical probability is also a very important open question.

It would be interesting to generalize this approach to systems driven by an external force (charge density waves, vortex lattices,...), and to study more subtle effects like those of temperature cycling. We would also like to point out that this route of OED seems to be a promising one towards a rigorous study of spin glasses. One should first obtain a rigorous derivation of the dynamical equations, and then understand the large time behaviour of these equations. This is certainly not easy, but it is a well defined mathematical problem and our work suggests that these coupled dynamical equations contain in some sense the full r.s.b. solution. A first step towards a rigorous derivation of the dynamical equations has been taken recently for the SK model 40]

\section{Acknowledgments}

We would like to thank J.P. Bouchaud, L. Cugliandolo, J. Hammann, J. Hertz, H. Kinzelbach, J. Kurchan, H. Horner, G.Parisi, E. Vincent and M.A. Virasoro for many interesting discussions.

\section{References}

[1] M. Mézard, G. Parisi, and M.A. Virasoro, "Spin glass theory and beyond", World Scientific (Singapore 1987);

[2] K. Binder and A.P. Young, Rev.Mod.Phys. 58 (1986) 801;

[3] K.H. Fischer, J.A. Hertz, "Spin Glasses" Cambridge University Press, (1991);

[4] L. Lundgren, P. Svedlindh, P. Nordblad and O. Beckman, Phys. Rev. Lett. 51 (1983) 911; P. Nordblad, P. Svedlindh, L. Lundgren, and L. Sandlund, Phys. Rev. B33 (1986) 645;

[5] R. V. Chamberlin, G. Mozurkevich and R. Orbach, Phys. Rev. Lett. 52 (1984) 867; R. Hoogerbets, Wei-Li Luo and R. Orbach, Phys. Rev. B34 (1986) 1719;

[6] M. Alba, J. Hamman, M. Ocio and Ph. Refrigier, J. Appl. Phys. 61 (1987) 3683; F. Lefloch, J. Hamman, M. Ocio and E. Vincent, Europhys. Lett. 18 (1992) 647; 
[7] M. Lederman, R. Orbach, J. M. Hamman, M. Ocio and E. Vincent Phys. Rev. B44 (1991) 7403; J. M. Hamman, M. Lederman, M. Ocio, R. Orbach and E. Vincent Physica A (1992) 278;

[8] G.J.M. Koper and H.J. Hilhorst, J. Phys. France 49 (1988) 429;

[9] D.S. Fisher and D.A. Huse, Phys. Rev. B38 (1988) 373;

[10] J. P. Bouchaud, J. Phys. I 2 (1992) 1705; J. P. Bouchaud, E. Vincent and J. Hamman, J. Phys. France 4 (1994) 139;

[11] C. DeDominicis, Phys.Rev. B18 (1978) 4913;

[12] H. Sompolinsky and A. Zippelius, Phys.Rev.Lett. 47 (1981) 359; Phys.Rev. B25 (1982) 6860;

[13] H. Sompolinsky, Phys.Rev.Lett. 47, 359 (1981);

[14] H. Horner, Z.Phys. B66 (1987) 175; M. Freixa-Pascual and H. Horner, Z.Phys. B80 (1990) 95;

[15] L.B. Ioffe,Phys. Rev. B38 (1988) 5181 ; V.S. Dotsenko, M.V. Feigelman and L.B. Ioffe, Soviet scientific reviews 15 (1990) 1;

[16] H. Eissfeller and M. Opper, Phys.Rev.Lett. 68 (1992) 2094;

[17] L.F. Cugliandolo and J. Kurchan, Phys.Rev.Lett.71 (1993) 173;

[18] H. Rieger, J. Phys. A 26 (1993) L615;

[19] L. Cugliandolo, J. Kurchan and F. Ritort, Evidence of Aging in Spin Glass Mean-Field Models, cond-mat/9307001 preprint (July 1993);

[20] E. Marinari and G. Parisi, J. Phys. A 26 (1993) L1149;

[21] T. Nattermann and P. Rujan, Int. J. Mod. Phys. B3 (1989) 1597; T. Natterman and J. Villain, Phase transitions 11 (1988) 5;

[22] M.V. Feigelman, V.B. Geshkenbein, A.I. Larkin and V.M. Vinokur, Phys.Rev.Lett. 63 (1989) 2303;

[23] J.P.Bouchaud, M.Mézard and J.S.Yedidia, Phys.Rev.Lett. 67(1991)3840 and Phys.Rev. B46(1992)14686;

[24] M.Kardar, G.Parisi and Y.C.Zhang, Phys.Rev.Lett. 56(1986)889;

[25] D. Fisher, M.P.A. Fisher, D. Huse, Phys. Rev. B 43, 130 (1991);

[26] M. Mézard and G. Parisi, J.Phys.A 23, L1229 (1990); J. Physique. I 1, 809 (1991); 
[27] J. Villain,B. Semeria, F. Lanon and L. Billard, J.Phys. C16, 2588 (1983); J. Villain J.Phys.A 21, L1099 (1988);

[28] A. Engel, J. Physique Lett. 46, L409 (1985);

[29] M. Mézard and G. Parisi, J.Phys. I France 2, 2231 (1992);

[30] A. Engel, Replica symmetry breaking in one dimension, Gottingen University preprint, 1993;

[31] S. Franz, M. Mézard, Off-equilibrium glassy dynamics: a simple case. LPTENS preprint 93/39, to be published in Europhysics Letters

[32] H. Kinzelbach and H. Horner, J.Phys. I France 3, 1329 (1993) ;

[33] H. Kinzelbach and H. Horner, J.Phys. I France 3, 1901 (1993);

[34] L. Cugliandolo, S. Franz, J. Kurchan and M. Mézard, in preparation;

[35] A. Crisanti, H. Horner and H-J Sommers, Z.Phys. B92 (1993) 257;

[36] T.R. Kirkpatrick and D. Thirumalai, Phys.Rev.Lett. 58 (1987) 2091, Phys.Rev. B38 (1987) 5388;

[37] L.F. Cugliandolo and J. Kurchan, On the out of equilibrium relaxation of the Sherrington Kirkpatrick model. Rome University preprint 977;

[38] M. Mézard, G. Parisi, N. Sourlas, G. Toulouse and M.A. Virasoro, Phys.Rev.Lett. 52 (1984) 1156; J. Physique 45 (1984) 843;

[39] J.O. Anderson, J. Mattson, and P. Svedlinh, Phys.Rev. 46 (1992) 8297;

[40] G. BenArous and A. Guionnet, Large deviations for Langevin spin glass dynamics, preprint Université de Paris Sud (mathématiques) 93.65; A. Guionnet, Large deviations for Langevin spin glass dynamics, part II, preprint Université de Paris Sud (mathématiques) 93.79.

\section{Appendix A}

In this appendix we sketch the derivation of the mean field dynamical equations (19) for the toy-model by the cavity method [1]. This method provides the same results as the functional derivation of [32]. We include a brief description here because it is maybe more explicit on the physical content of the derivation. Apart from unessential complications, the derivation could be done similarly for the more general equations (77,8,9) for finite $D$. The method involves an induction over the number dimensions $N$ of the space in which the particle lives, together with a large $N$ limit. We pass from a $N$ dimensional system described by $\phi=\left\{\phi_{1}, \ldots, \phi_{N}\right\}$ to a $N+1$ dimensional one described by $\phi$, plus a new component $\phi_{0}$. In the derivation we follow 
a procedure analogous to that which has been used e.g. to study the statics and the equilibrium dynamics of the SK model. We will make crucial use of two hypotheses that mutatis mutandis habe been put forward in that case. Namely, the applicability of the linear response theory fort the Langevin equation (LRT), and the fact that the responses $\delta \phi_{\alpha}(t) / \delta \eta_{\beta}(s)$ can be considered small (in a suitable sense) for $\alpha \neq \beta$. A justification of these in the case of equilibrium dynamics is given in [1]. For OED, we just assume these two facts. It will be interesting to see if similar assumptions are contained in the functional approach, or whether these facts can be derived.

Consider the Langevin equation for the toy-model:

$$
\begin{gathered}
\frac{\mathrm{d} \phi_{\alpha}(t)}{\mathrm{d} t}=-\frac{\partial H(\phi(t))}{\partial \phi_{a}}+\eta_{\alpha}(t) \\
\left\langle\eta_{\alpha}(t) \eta_{\beta}(s)\right\rangle=2 T \delta_{\alpha \beta} \delta(t-s) .
\end{gathered}
$$

If an infinitesimal perturbation $\delta H(\phi)$ is added to $H$, the perturbed process $\phi^{*}(t)$ can be expressed in terms of the unperturbed one $\phi(t)$ by the linear response relation:

$$
\phi_{\alpha}^{*}(t)=\phi_{\alpha}(t)-\sum_{\beta} \int_{0}^{t} \mathrm{~d} s \frac{\partial \delta H(\phi(s))}{\partial \phi_{\beta}} \frac{\delta \phi_{\alpha}(t)}{\delta \eta_{\beta}(s)} .
$$

Let us now introduce the new component, and denote by $V_{N}(\phi)$ and $V_{N+1}\left(\phi_{0}, \phi\right)$ the random potentials for the $N$ and $N+1$ components systems respectively. In making this step, the Hamiltonian $H=\mu \phi^{2} / 2+V_{N}(\phi)$ will undergo the variation

$$
\begin{array}{r}
\delta H\left(\phi_{0}, \boldsymbol{\phi}\right)=\mu \phi_{0}^{2} / 2+\delta V\left(\phi_{0}, \boldsymbol{\phi}\right) \\
\delta V\left(\phi_{0}, \boldsymbol{\phi}\right)=V_{N+1}\left(\phi_{0}, \boldsymbol{\phi}\right)-V_{N}(\boldsymbol{\phi}) .
\end{array}
$$

The $\phi_{\alpha}^{*}$ and $\phi_{\alpha}$ in (47) have to be identified with the $\alpha$-th component of the position of the particle respectively in presence and in absence of $\phi_{0}$.

To study the statistical properties of $\delta V$ we can expand in series the correlations of the potential of the $N+1$ components system

$$
\overline{V_{N+1}\left(\phi_{0}, \phi\right) V_{N+1}\left(\psi_{0}, \boldsymbol{\psi}\right)}=-(N+1) f\left(\frac{\left[(\boldsymbol{\phi}-\boldsymbol{\psi})^{2}+\left(\phi_{0}-\psi_{0}\right)^{2}\right]}{(N+1)}\right)
$$

and retain only the terms of the series which do not tend to zero when $N \rightarrow \infty$.

In this way we find:

$$
\begin{aligned}
\overline{\delta V\left(\phi_{0}, \boldsymbol{\phi}\right) \delta V\left(\psi_{0}, \boldsymbol{\psi}\right)}= & -\left\{f\left((\boldsymbol{\phi}-\boldsymbol{\psi})^{2} / N\right)-\frac{(\boldsymbol{\phi}-\boldsymbol{\psi})^{2}}{N} f^{\prime}\left((\boldsymbol{\phi}-\boldsymbol{\psi})^{2} / N\right)\right. \\
& \left.+\left(\phi_{0}-\psi_{0}\right)^{2} f^{\prime}\left((\boldsymbol{\phi}-\boldsymbol{\psi})^{2} / N\right)\right\}
\end{aligned}
$$


These formulas can be obtained expanding formally $V_{N+1}\left(\phi_{0}, \boldsymbol{\phi}\right)$ in powers of $\phi_{0}$ up to the second order. In this way, denoting $b=(\boldsymbol{\phi}-\boldsymbol{\psi})^{2} / N$, one easily shows that $\delta V\left(\phi_{0}, \phi\right)$ can be written as

$$
\delta V\left(\phi_{0}, \boldsymbol{\phi}\right)=A(\boldsymbol{\phi})+B(\boldsymbol{\phi}) \phi_{0}+D(\boldsymbol{\phi}) \phi_{0}^{2}
$$

where $A, B$ and $D$ are gaussian random functions with zero averages and correlations:

$$
\begin{aligned}
& \overline{A(\boldsymbol{\phi}) A(\boldsymbol{\psi})}=-\left[f(b)-b f^{\prime}(b)\right] \\
& \overline{A(\boldsymbol{\phi}) B(\boldsymbol{\psi})}=O(1 / N) \\
& \overline{A(\phi) D(\boldsymbol{\psi})}=-f^{\prime}(b) \\
& \overline{B(\phi) B(\boldsymbol{\psi})}=2 f^{\prime}(b) \\
& \overline{B(\boldsymbol{\phi}) D(\boldsymbol{\psi})}=O(1 / N) \\
& \overline{D(\boldsymbol{\phi}) D(\boldsymbol{\psi})}=O(1 / N)
\end{aligned}
$$

We can now write the Langevin equation for the zeroth component $\phi_{0}$

$$
\frac{\mathrm{d} \phi_{0}(t)}{\mathrm{d} t}=-\mu \phi_{0}-B\left(\phi^{*}(t)\right)-2 D\left(\phi^{*}(t)\right) \phi_{0}(t)+\eta_{0}(t)
$$

Using the LRT we find

$$
\begin{array}{cc}
B\left(\phi^{*}(t)\right) \quad & =B(\phi(t))-\int_{0}^{t} d s \sum_{\alpha \beta} \frac{\partial B(\phi(t))}{\partial \phi_{\alpha}} \frac{\delta \phi_{\alpha}(t)}{\delta \eta_{\beta}(s)} \\
\frac{\partial}{\partial \phi_{\beta}}[ & {\left[(\phi(s))+B(\phi(s)) \phi_{0}(s)+D(\phi(s)) \phi_{0}^{2}(s)\right]} \\
D\left(\phi^{*}(t)\right) \quad=D(\phi(t))-\int_{0}^{t} d s \sum_{\alpha \beta} \frac{\partial D(\phi(t))}{\partial \phi_{\alpha}} \frac{\delta \phi_{\alpha}(t)}{\delta \eta_{\beta}(s)} \\
\frac{\partial}{\partial \phi_{\beta}}\left[A(\phi(s))+B(\phi(s)) \phi_{0}(s)+D(\phi(s)) \phi_{0}^{2}(s)\right] .
\end{array}
$$

At this point we use the hypothesis that $\delta \phi_{\alpha}(t) / \delta \eta_{\beta}(s)$ is small for $\alpha \neq \beta$. More precisely we suppose that as in the SK mode $1 / N^{2} \sum_{\alpha \beta} \delta \phi_{\alpha}(t) / \delta \eta_{\beta}(s)$ and analogous sums will tend to zero in the large $N$ limit. One deduces that (54) reads in this limit:

$$
\begin{aligned}
B\left(\phi^{*}(t)\right) & =B(\phi(t))-\int_{0}^{t} \mathrm{~d} s 4 f^{\prime \prime}(b(t, s)) r(t, s) \phi_{0}(s) \\
D\left(\phi^{*}(t)\right) & =D(\phi(t))-\int_{0}^{t} \mathrm{~d} s 2 f^{\prime \prime}(b(t, s)) r(t, s) .
\end{aligned}
$$

where $b(t, s)=(\phi(t)-\phi(s))^{2} / N$ and $r(t, s)=(1 / N) \sum_{\alpha}\left(\delta \phi_{a}(t) / \delta \eta_{a}(s)\right)$.

Denoting $m(t, s)=4 f^{\prime \prime}(b(t, s)) r(t, s)$ and making use of (55) the Langevin equation (53) is rewritten as

$\frac{\mathrm{d} \phi_{0}(t)}{\mathrm{d} t}=-\mu \phi_{0}(t)-B(\phi(t))-2 D(\phi(t)) \phi_{0}(t)+\int_{0}^{t} \mathrm{~d} s m(t, s)\left[\phi_{0}(t)-\phi_{0}(s)\right]+\eta_{0}(t)$. 
(In deriving (56) we have dropped a term proportional to $D(\phi(t)) \phi_{0}(t)$ which is negligible because of the vanishing correlations (52) of $D$ at large $N$.) The term $B(\phi(t))$ is a random field with zero mean and correlations $\overline{B(\phi(t)) B(\phi(t))}=2 f^{\prime}(b(t, s))=w(t, s)$. Therefore equation (56) is the usual Langevin equation on one single component, with a condition of selfconsistence, from which the dynamical equations are easily derived. We just notice that this derivation shows a property of self averageness of the response, namely the fact that the response function of each component is identical: $r(t, s)=<\delta \phi_{0}(t) / \delta \eta_{0}(s)>$ 


\section{Figure Captions}

Fig. 1: a) An estimate of the large time limit, $C_{\infty}$, of the autocorrelation $\mathrm{C}(\mathrm{t}, \mathrm{t})$, from the numerical solution of the dynamical equations with a grid size $h=1.2$. The derivative $d C(t, t) / d t$ has been fitted to a power law with logarithmic corrections (see text). For each time $t, C_{\infty}$ is approximated by $C(t, t)$ plus the integral of the fit of the derivative. The plot gives this estimation, versus the time $t$. The analytical result from r.s.b., $C_{\infty}=21.5$, is compatible with the result, when one takes into account the effects due to the finite value of $h$ (see Fig. 1b) and to the uncertainties of the fit. b) The difference between $C(t, t)$ computed with a grid size $h=1.2$ and that computed with $h=.8$, plotted versus time in a Log-Log plot. This difference seems to extrapolate to zero at large times (with a power law behaviour).

Fig. 2: a) An estimate of the large time limit , $E_{\infty}$, of the energy $E(t)$, from the numerical solution of the dynamical equations with a grid size $h=1.2$. The procedure is the same as that followed for the estimate of $C(t, t)$ in Fig.1. The analytic result from r.s.b., $E_{\infty}=-1.3660$, is compatible with this data when one takes into account the effect of the extrapolation to $h=0$ (see Fig. 2b, and the text). b)The difference between $E(t)$ computed with a grid size $h=1.2$ and that computed with $h=.8$, plotted versus time. This difference is well approximated by a power law fit with an asymptote equal to .0116.

Fig. 3: The quantities $A(t)$ (continuous line) and $B(t)$ (dotted line) defined in the text in a Log-Log scale. $A(t)$ is better approximated by a power law then $B(t)$. A pure power law fit on the last 300 points over a total of 890 gives $A(t)=.04+14.8 t^{-.57}$ with a relative error on the whole interval of the order $\Delta A / A \sim 10^{-6}$ and $B(t)=.29+35.1 t^{-.57}$ with $\Delta B / B \sim 10^{-5}$.

Fig. 4: In a), the response $r\left(t_{w}+\tau, t_{w}\right)$ versus $\tau$. From top to bottom, $t_{w}=432,504,576,648$. Also shown (bottom curve) is the power law extrapolation of these curves to $t_{w} \rightarrow \infty$, together with the prediction for $r_{a s}(\tau)$ from the asymptotic dynamics (these last two curves are nearly undistinguishable). In b), similar curves for the corrrelation $b\left(t_{w}+\tau, t_{w}\right)$. From top to bottom, $t_{w}=432,504,576,648$, the extrapolation and the expected result from the asymptotic dynamics.

Fig. 5: The thermoremanent magnetization $\mathcal{M}\left(\tau, t_{w}\right)$ defined in (44) versus $\tau$ for fixed values of the waiting time $t_{w}$, on logarithmic scales. From bottom to top, $t_{w}=38.4,76.8,153.6,307.2,614.4$.

Fig. 6: The normalised correlation $C\left(t_{w}+\tau, t_{w}\right) / C\left(t_{w}, t_{w}\right)$ versus $\tau$, for fixed values of the waiting time $t_{w}$. From bottom to top, $t_{w}=38.4$, $76.8,153.6,307.2,614.4$. 
Fig. 7: The third dynamical moment $\mathcal{C}_{3}(t)$, defined in 40 versus $t$. The inset is a log-log plot of the relative difference between the moment at time $t$ and the prediction from the scenario of hierarchical crossovers concerning its large time behaviour: $\left(9011-\mathcal{C}_{3}(t)\right) / 9011$ versus $t$.

Fig. 8: The same plot as in Fig. 7, for the fifth dynamical moment versus time, and its convergence to the theoretical result $5.7610^{6}$.

Fig. 9: The function $C\left(t, t^{\prime}\right) / C(t, t)$ versus the time $t$, along lines in the $t, t^{\prime}$ plane such that the f.d. ratio $U\left(t, t^{\prime}\right)$ is constant, equal to $u_{0}$. From bottom to top, $u_{0}=.1, .2, \ldots, .9$. If the scenario of hierarchical crossovers would hold, at infinite $t$ the curves with $u 0<.375$ should extrapolate to .744 , the ones with $u_{0}>.411$ should extrapolate to .859 . There is no such indication on this time scale. 